D

Dept of Respiratory Medicine, AJ Institute of Medical Sciences, Mangalore, Karnataka.

\title{
A middle-aged man with generalised weakness and dyspnoea
}

\section{Case report}

A 50-year-old male presented with progressive breathlessness and generalised muscular weakness for the last 10 days. He felt extremely weak and could barely walk due to breathlessness. His dyspnoea was increased when lying down and decreased by an upright posture. He had no other respiratory or cardiac symptoms.

He had no fever, or gastrointestinal or urinary symptoms. He denied having any neurological or psychiatric symptoms. There was no past history of any respiratory or cardiac disease.

\section{Task 1}

Which of the following is the least likely cause of breathlessness in this patient?
a. Acute exacerbation of asthma
b. Metabolic acidosis
c. Respiratory muscle dysfunction due to neuromuscular diseases
d. Severe anaemia
e. Electrolyte imbalance

Cite as: Sharma MV, Shekh IA. A middle-aged man with generalised weakness and dyspnoea. Breathe 2017; 13: $217-224$ 


\section{Answer 1}

a. Acute exacerbation of asthma. He had no respiratory symptoms in the past and no other symptoms suggestive of asthma. Generalised muscular weakness associated with breathlessness is indicative of a systemic or neuromuscular disease. This can occur in a variety of rapidly progressive neuromuscular diseases with respiratory muscle involvement. Metabolic disorders like uncontrolled diabetes mellitus with ketoacidosis, renal disease with acidosis and other metabolic conditions leading to acidosis can also present with generalised weakness with breathlessness. Electrolyte imbalance, such as severe hyponatraemia, hypocalcaemia and hypokalaemia, can also lead to similar symptoms. Severe anaemia is common in developing countries and can present with these symptoms when there is subacute blood loss.

He had no other symptoms or predisposing factors for anaemia. He had no history of liver disease, renal disease or diabetes mellitus. He had no other illness in the past that could predispose to the development of an electrolyte imbalance.

On further enquiry, he admitted having intermittent episodes of muscle weakness with drooping of the eyelids, which was relieved without medication for the last 5 years. He did not have any visual disturbances or headache. However, he did not complain about drooping of the eyelids at the time of hospitalisation. He had consulted two ophthalmologists, two physicians and one neurosurgeon with these symptoms in the last 5 years. No definitive diagnosis was made. He had no other sensory or motor symptoms.

\section{Task 2}

What could be the cause of his symptoms, with this history?
a. Multiple sclerosis
b. Myasthenia gravis
c. Chronic hypocalcaemia
d. Lambert-Eaton syndrome
e. Pituitary tumour 


\section{Answer 2}

b. Myasthenia gravis. Ocular symptoms, muscular weakness and symptoms of respiratory muscle dysfunction are diagnostic of myasthenia gravis [1].

He had no other ocular symptoms. He had occasional difficulty swallowing over the last 4 weeks but no difficulty chewing. He had no history of fatigue on exercise prior to 4 weeks. In myasthenia gravis, symptoms include extraocular muscle weakness, ptosis and bulbar muscle weakness. Muscular weakness worsens as the day progresses. Symptoms may remain limited to the extraocular and eyelid muscles for years. Limb weakness is usually more severe proximally than distally in myasthenia gravis [1].
His respiratory rate was 34 breaths per min. His oxygen saturation while breathing room air on arrival was $86 \%$. His accessory muscles of respiration were active. His heart rate was 126 beats per min and regular.

\section{Task 3}

Which of the following is not a sign of respiratory muscle dysfunction?

a. Inability to take a deep inspiration

b. Reduced breath holding time

c. Inability to clear airway secretions by coughing

d. Dyspnoea relieved by lying down

e. Inability to speak properly 


\section{Answer 3}

d. Dyspnoea relieved by lying down. In respiratory muscle dysfunction, dyspnoea will worsen on lying down as the abdominal contents will move up and further hamper the diaphragmatic movement.

On examination, he was unable to take a deep breath and his voice was feeble. His breath holding time was $<10 \mathrm{~s}$. He could not cough properly. There was no other abnormality on cardiorespiratory system examination. All these findings are indicative of respiratory muscle dysfunction. He had grade 3 power in both upper and lower limbs. No other neurological abnormality was found. He had no ocular signs. All these findings are suggestive of myasthenia gravis with predominant involvement of respiratory muscles [1].

Ocular symptoms are the most common presenting symptom in myasthenia gravis [2]. In $>50 \%$ of patients who develop myasthenia gravis, the first signs and symptoms involve the eye. The patient may have drooping of one or both eyelids (ptosis) and double vision (diplopia), which may be horizontal or vertical. Diplopia may improve or resolve when one eye is closed. Ptosis in myasthenia can be transient or persistent. It can improve without treatment in some cases [3]. Waxing and waning of symptoms do occur in myasthenia gravis [3].

Some patients with myasthenia gravis develop deterioration of muscle weakness, which may be rapid, leading to respiratory failure. This patient had isolated respiratory muscle involvement, which is a rare manifestation of myasthenia gravis [4].

\section{Task 4}

Which of the following is least likely to occur in patients with neuromuscular disease causing respiratory muscle weakness?

a. Inability to swallow properly

b. Drooling of saliva

c. Profound weakness of neck muscles

d. Recurrent respiratory tract infections

e. Hypoxic respiratory failure 


\section{Answer 4}

e. Hypoxic respiratory failure. Respiratory muscle weakness leads to hypercapnic respiratory failure due to hypoventilation.

The signs of impending respiratory failure due to neuromuscular disease are [5]:

- Tachypnoea and sinus tachycardia

- Staccato speech

- Inability to count up to 20

- Profound weakness of neck flexion

- Use of accessory muscles (visible)
- Orthopnoea

- Paradoxical breathing pattern

- Signs of bulbar dysfunction (nasal voice, accumulation of saliva and weak cough)

\section{Task 5}

Which of the following tests is not useful to assess respiratory muscle function?
a. Maximal inspiratory pressure
b. Spirometry
c. Maximal expiratory pressure
d. Maximal sniff pressures
e. Transdiaphragmatic pressure 


\section{Answer}

b. Spirometry. Spirometry can be abnormal in respiratory muscle dysfunction but is not useful to assess respiratory muscle function. In respiratory muscle dysfunction, spirometry may show a restrictive abnormality with increased residual volume. A fall in vital capacity (VC) between the upright and supine positions has been used to assess diaphragm weakness. Normally, there is $<10 \%$ drop in VC when changing from the upright to the supine position. With bilateral diaphragm paralysis, VC may be reduced by $>30 \%$ in the supine position [6]

We could not perform spirometry in this patient as he was too breathless and hypoxic to perform the test. The Tensilon test was performed in the emergency department, which confirmed myasthenia gravis.

This patient was immediately started on pyridostigmine (60 $\mathrm{mg}$ four times daily) and prednisolone (60 $\mathrm{mg}$ per day). He showed marked improvement within hours of initiation of pyridostigmine. He was on noninvasive ventilation (NIV) for $24 \mathrm{~h}$. His dyspnoea and generalised weakness relieved, and he was disconnected from NIV after 24 h.

\section{Task 6}

1. Which of the following statements regarding the thymus is correct in myasthenia gravis?

a. Thymic tumour is more common than thymic hyperplasia

b. Thymectomy should be performed in all patients with an enlarged thymus

c. Emergency thymectomy should be performed in myasthenic crisis

d. Thymectomy improves symptoms in all patients

e. Thymectomy gives immediate relief to symptoms

2. Which of the following statements regarding computed tomography (CT) of the thorax is incorrect in myasthenia gravis?

a. It should be performed in all patients

b. It is sensitive to the detection of thymic lesions

c. It is useful to rule out other intrathoracic malignancy

d. It is not required if chest radiography is normal

e. It can be normal 


\section{Answer 6}

1. b. Thymectomy should be performed in all patients with an enlarged thymus. Thymic hyperplasia is more common than thymic tumour in patients with myasthenia gravis. Emergency thymectomy should not be performed in myasthenic crisis as the mortality is high if the patient is operated upon during myasthenic crisis. Thymectomy improves symptoms in majority of patients with thymic lesions but not in all patients. After thymectomy, it takes few weeks to months for improvement of symptoms [7].

2. d. A CT scan of chest should be performed in all in myasthenia gravis patients as CT is sensitive to detect thymic lesions and useful to rule out intrathoracic malignancy [7].

A chest CT scan was performed in this patient (figure 1). It was normal.

Serum acetylcholine receptor (AChR) binding antibody concentration was $1.17 \mathrm{nmol} \cdot \mathrm{L}^{-1}$, confirming diagnosis of myasthenia gravis (normal range 0.00-0.25 $\mathrm{nmol} \cdot \mathrm{L}^{-1}$ ). Normally, no AChR binding antibody is present in the bloodstream. AChR binding antibody is the most common antibody found in myasthenia gravis patients [8]. Binding and blocking antibodies together have high sensitivity and specificity (99.6\%) for myasthenia gravis patients. Serum AChR binding antibody confirms the diagnosis of myasthenia gravis. It is useful to distinguish acquired myasthenia gravis ( $90 \%$ positive) from congenital disease (AChR negative). It is also useful in detecting subclinical myasthenia gravis in patients with thymoma. It can be used to monitor disease progression in myasthenia gravis or response to immunotherapy.

Myasthenia gravis is a chronic autoimmune neuromuscular disease characterised by varying degrees of weakness of the skeletal (voluntary) muscles of the body. It is caused by a defect in the transmission of nerve impulses to muscles. The thymus may give incorrect instructions to developing immune cells, resulting in autoimmunity and the production of the AChR antibodies [9]. Antibodies block, alter or destroy the receptors for acetylcholine at the neuromuscular junction, which prevents muscle contraction from occurring. AntiAChR binding antibody level may be low at the onset of myasthenia gravis and gradually become elevated in a late stage. It is worthwhile to repeat test when initial values normal.
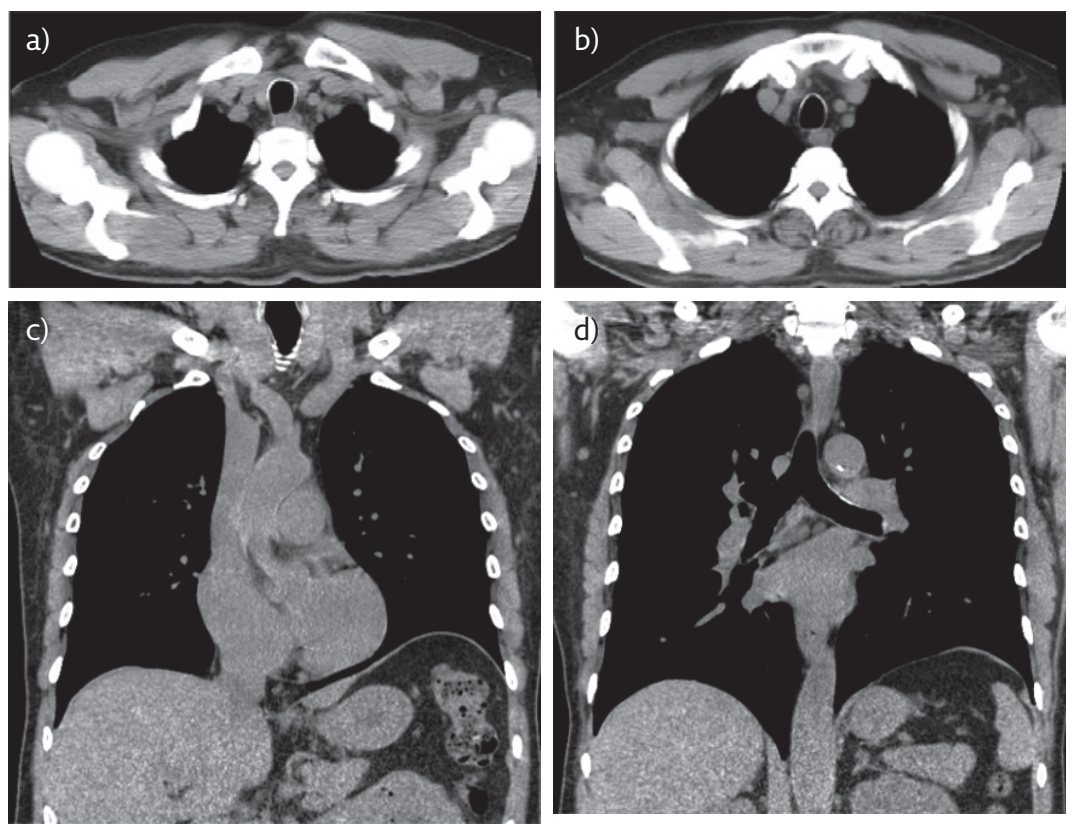

Figure 1 Computed tomography images.

Medications that can increase myasthenia symptoms are:

- $\beta$-blockers

- Quinidine gluconate, quinidine sulfate and quinine

- Phenytoin, certain anaesthetics and some antibiotics

Tests that help to confirm the diagnosis of myasthenia gravis include AChR binding antibodies, the edrophonium test and electromyography [8]. Medical treatment includes anticholinesterase agents, plasmapheresis and various immunosuppressive drugs. Surgical treatment may include removal of the thymus.

Task 7

Which of the following statements regarding Lambert-Eaton syndrome is incorrect?

a. Deep tendon reflexes are decreased or absent

b. Muscular weakness decreases after contractions

c. Ocular symptoms are rare

d. The most common cause is oat cell carcinoma of lung

e. Muscular weakness affects the arms more than the legs 


\section{Answer 7}

e. Myasthenia gravis should be differentiated from Lambert-Eaton syndrome [10]. Deep tendon reflexes are decreased or absent in Lambert-Eaton syndrome but normal in myasthenia gravis. In Lambert-Eaton syndrome, muscular weakness affects the legs more than the arms. This condition is due to antibodies against voltage-gated calcium channels. Lambert-Eaton syndrome is treated with 3,4-diaminopyridine, which blocks potassium channels in the distal motor terminal.
The patient was discharged after 4 days in a stable clinical condition with oral medication.

The prognosis of myasthenia gravis is variable, ranging from complete remission ( $50 \%$ with thymus surgery), to patients who lead relatively normal lives with continued treatment, to a poor prognosis as the disease advances.

\section{Learning points}

Consider neuromuscular diseases that cause respiratory muscle weakness as a cause of dyspnoea, especially when there is no obvious cause of the dyspnoea. Careful history taking and physical examination is helpful in these cases. Sometimes, patients with myasthenia gravis can present with respiratory failure as the predominant symptom.

\section{Conflict of interest}

None declared.

\section{References}

1. Gilhus NE, Verschuuren JJ. Myasthenia gravis: subgroup classification and therapeutic strategies. Lancet Neurol 2015; 14: 1023-1036.

2. Sieb JP. Myasthenia gravis: an update for the clinician. Clin Exp Immunol 2014; 175: 408-418.

3. KeeseyJC. Clinical evaluation and management of myasthenia gravis. Muscle Nerve 2004; 29: 484-505.

4. Dushay KM, Zibra JD, Jensen WA. Myasthenia gravis presenting as isolated respiratory failure. Chest 1990; 97: 232-234.

5. Perrin C, Unterborn JN, D’Ambrosio C, et al. Pulmonary complications of chronic neuromuscular diseases and their management. Muscle Nerve 2004; 29: 5-27.
6. Ward NS, Hill NS. Pulmonary function testing in neuromuscular disease. Clin Chest Med 2001; 22: 769-781.

7. Takanami I, Abiko T, Koizumi S. Therapeutic outcomes in thymectomied patients with myasthenia gravis. Ann Thorac Cardiovasc Surg 2009; 15: 373-377.

8. Benatar M. A systematic review of diagnostic studies in myasthenia gravis. Neuromuscul Disord 2006; 16: 459-467.

9. Grob D, Brunner N, Namba T, et al. Lifetime course of myasthenia gravis. Muscle Nerve 2008; 37: 141-149.

10. Pascuzzi RM. Pearls and pitfalls in the diagnosis and management of neuromuscular junction disorders. Semin Neurol 2001; 21: 425-440 